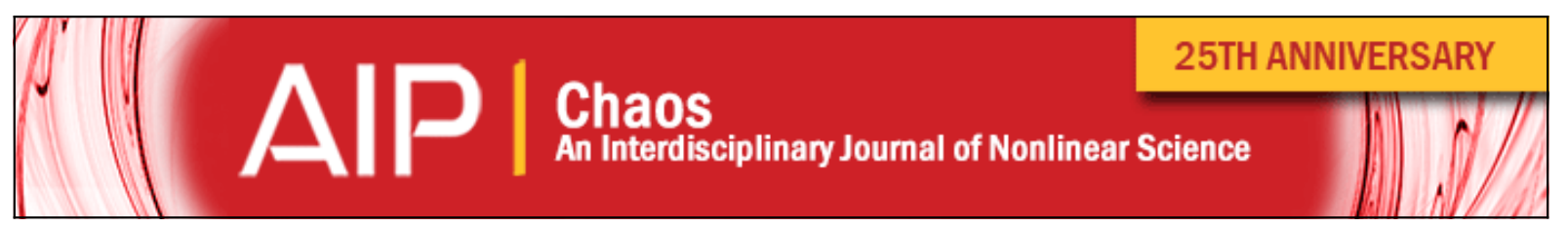

\title{
A chimeric path to neuronal synchronization
}

Easwara Moorthy Essaki Arumugam and Mark L. Spano

Citation: Chaos: An Interdisciplinary Journal of Nonlinear Science 25, 013107 (2015); doi: 10.1063/1.4905856

View online: http://dx.doi.org/10.1063/1.4905856

View Table of Contents: http://scitation.aip.org/content/aip/journal/chaos/25/1?ver=pdfcov

Published by the AIP Publishing

\section{Articles you may be interested in}

Phase synchronization analysis of prefrontal tissue oxyhemoglobin oscillations in elderly subjects with cerebral infarction

Med. Phys. 41, 102702 (2014); 10.1118/1.4896113

Impact of delays on the synchronization transitions of modular neuronal networks with hybrid synapses

Chaos 23, 033121 (2013); 10.1063/1.4817607

Synchronization analysis of voltage-sensitive dye imaging during focal seizures in the rat neocortex

Chaos 21, 047506 (2011); 10.1063/1.3640043

Intermittent synchronization in a network of bursting neurons

Chaos 21, 033125 (2011); 10.1063/1.3633078

Mass synchronization: Occurrence and its control with possible applications to brain dynamics

Chaos 20, 045106 (2010); 10.1063/1.3527993

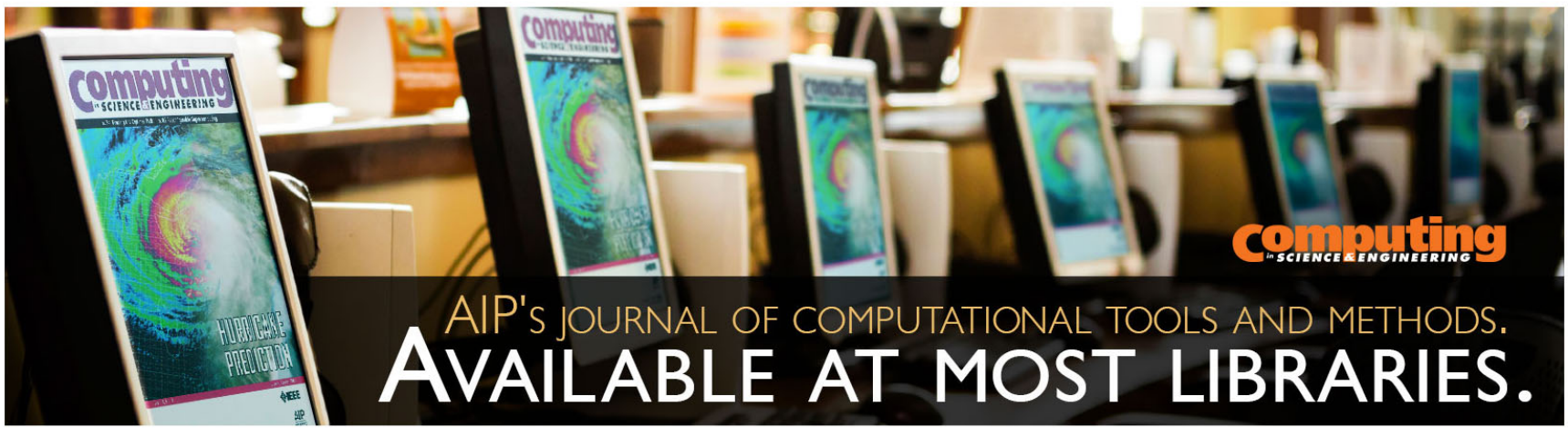




\title{
A chimeric path to neuronal synchronization
}

\author{
Easwara Moorthy Essaki Arumugam ${ }^{\text {a) }}$ and Mark L. Spano \\ School of Biological and Health Systems Engineering, Arizona State University, Tempe, Arizona 85287-9709, \\ USA
}

(Received 18 August 2014; accepted 15 December 2014; published online 9 January 2015)

\begin{abstract}
Synchronization of neuronal activity is associated with neurological disorders such as epilepsy. This process of neuronal synchronization is not fully understood. To further our understanding, we have experimentally studied the progression of this synchronization from normal neuronal firing to full synchronization. We implemented nine FitzHugh-Nagumo neurons (a simplified HodgkinHuxley model) via discrete electronics. For different coupling parameters (synaptic strengths), the neurons in the ring were either unsynchronized or completely synchronized when locally coupled in a ring. When a single long-range connection (nonlocal coupling) was introduced, an intermediate state known as a chimera appeared. The results indicate that (1) epilepsy is likely not only a dynamical disease but also a topological disease, strongly tied to the connectivity of the underlying network of neurons, and (2) the synchronization process in epilepsy may not be an "all or none" phenomenon, but can pass through an intermediate stage (chimera). (C) 2015 AIP Publishing LLC.
\end{abstract}

[http://dx.doi.org/10.1063/1.4905856]

Synchronization is a universal phenomenon observed in natural systems ranging from mechanical oscillators to hormonal cycles in the body to the synchronized flashing seen in fireflies. In the brain, synchronization is often associated with epileptiform behavior. Epilepsy, one of the most common neurological disorders, can affect memory, motor function, and consciousness. It is associated with synchronous discharge of the neurons in a brain region termed the epileptic focus. ${ }^{1}$ This collective firing (observed in EEG as interictal spikes) is usually considered to arise suddenly and spontaneously. However, recent theoretical ${ }^{2,3}$ and experimental ${ }^{4,5}$ results from nonlinear dynamics suggest that the process of synchronization can be a more complex phenomenon. Here, we explore the two hypotheses that such synchronization may be caused by the connectivity of the underlying network of neurons and that the synchronization need not be all or none, but alternatively may pass through an extended, partially synchronized state (chimera). Experimentally, we employ a physical network of nine electronic Fitzhugh-Nagumo neurons ${ }^{6,7}$ arranged in a ring, modified by introduction of a single, weak, and longrange interaction. Advantages to this approach are that the "neurons" are actual physical constructs, with all the imprecision and noisiness which that implies, and the interconnections between the neurons are under our control.

\section{INTRODUCTION}

The Fitzhugh-Nagumo description ${ }^{6,7}$ of the neuron, based on the seminal work of Hodgkin and Huxley, ${ }^{8}$ captures the fundamental excitability and oscillatory dynamics of a neuron ${ }^{9}$ without requiring the complexity of the full Hodgkin-Huxley model.

We employ the equations used by Izhikevich ${ }^{10}$

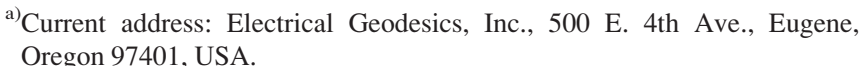

$$
\begin{aligned}
& \dot{V}_{i}=V_{i}\left(a-V_{i}\right)\left(V_{i}-1\right)-w_{i}+I+\sum_{j=1 ; j \neq i}^{j=N} G_{i j}\left(V_{j}-V_{i}\right), \\
& \dot{w}_{i}=b V_{i}-c w_{i},
\end{aligned}
$$

where $V_{i}$ is the membrane voltage and $w_{i}$ is a recovery variable mimicking the activation of an outward current. The constant $a$ describes the overall shape of the voltage curve, while the constants $b \geq 0$ and $c \geq 0$ describe the recovery variable's kinetics. The current $I$ represents injected current and is set to zero for this experiment. The neurons are coupled to each other via coupling strengths $G_{i j}$. The subscript $i(1 \leq i \leq N)$ indexes the neurons in the chain.

As shown in Fig. 1(a), we connected 9 neurons in a ring and, for the initial part of this paper, we chose to make the coupling symmetrical. Thus, $G_{i j}=G_{j i} \equiv G$ only if $j=i \pm 1$, otherwise zero. To complete the ring, we must also add the connection $G_{19}=G_{91} \equiv G$. The detailed electronic implementation is detailed below. This is similar to the experiment of Gambuzza et al. ${ }^{11}$ in that we use a ring of electronic Fitzhugh-Nagumo neurons; however we constructed the symmetry-broken ring with an odd number of neurons and a single small-world-type link with weak coupling. Additionally their inter-neuron coupling is accomplished via the recovery variable, while we couple the neurons via the voltage variable.

\section{EXPRESSION OF THE FITZHUGH-NAGUMO NEURON IN DISCRETE ELECTRONICS}

For the purposes of implementing these neurons, it is useful to rewrite Eqs. (1) as

$$
\begin{aligned}
& \dot{V}_{i}=-V_{i}^{3}+V_{i}^{2}+a\left(V_{i}^{2}-V_{i}\right)-w_{i}+I+\sum_{j=1 ; j \neq i}^{j=N} G_{i j}\left(V_{j}-V_{i}\right), \\
& \dot{w}_{i}=b V_{i}-c w_{i} .
\end{aligned}
$$



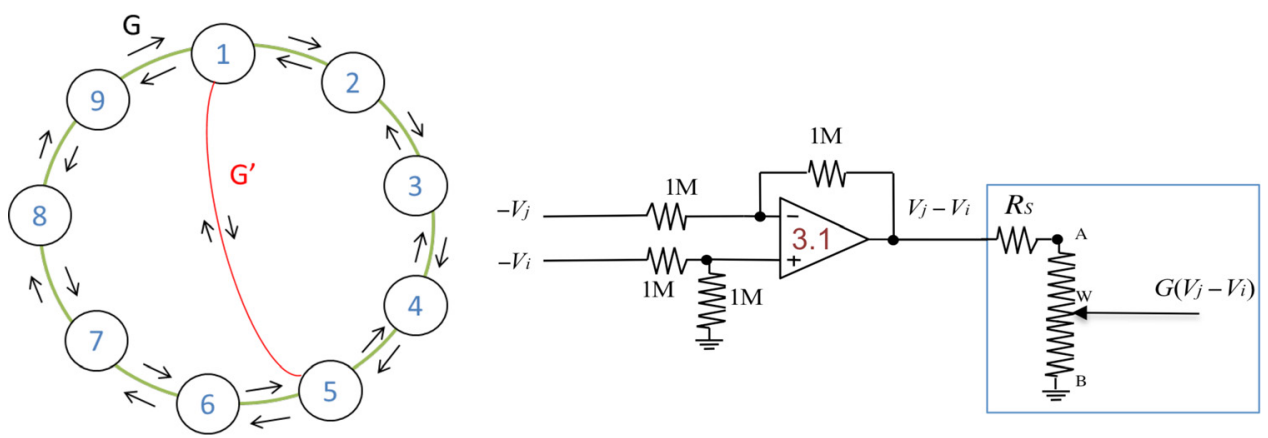

FIG. 1. Neuronal coupling. (a) Overall schematic of nonlocal coupling. As a control, all nine neurons were connected in a ring (bidirectional coupling) with the same coupling strength, $G=0.0087$. For testing our hypothesis, an additional connection from neuron 1 to neuron 5 was added, with coupling strength $G^{\prime}=0.000169$; other parameters are $a \sim$ threshold value between periodic firing and not firing (varies slightly by neuron), $b=0.32, c=0.32, I=0$. (b) Coupling circuit using an op-amp (3.1) as a subtractor feeding into a voltage divider with a resistor and a digital potentiometer.

The circuit implementing Eqs. (2) for a single neuron is shown in Fig. 2. Begin at the upper left, where a voltage $-V$ is introduced. (For clarity, we drop the subscript $i$ indexing the different neurons.) The voltage is passed through op amp (1.1) to change the sign of the voltage. Then the voltage is introduced into the two positive terminals of an Analog Devices 734 high speed, four-quadrant analog multiplier with a $100 \mathrm{MHz}$ bandwidth. The output is the product of the two input voltages scaled down by a factor of 10 . Thus, the output from the multiplier labeled (A) is $V^{2} / 10$. This voltage is then scaled back up by a factor of 10 by passing it through op amp (1.2). (Op amps add/subtract as well as rescale. The scaling is determined by the values of the nearby resistors. Here, we use the Analog Devices 704 quad op amp.) The

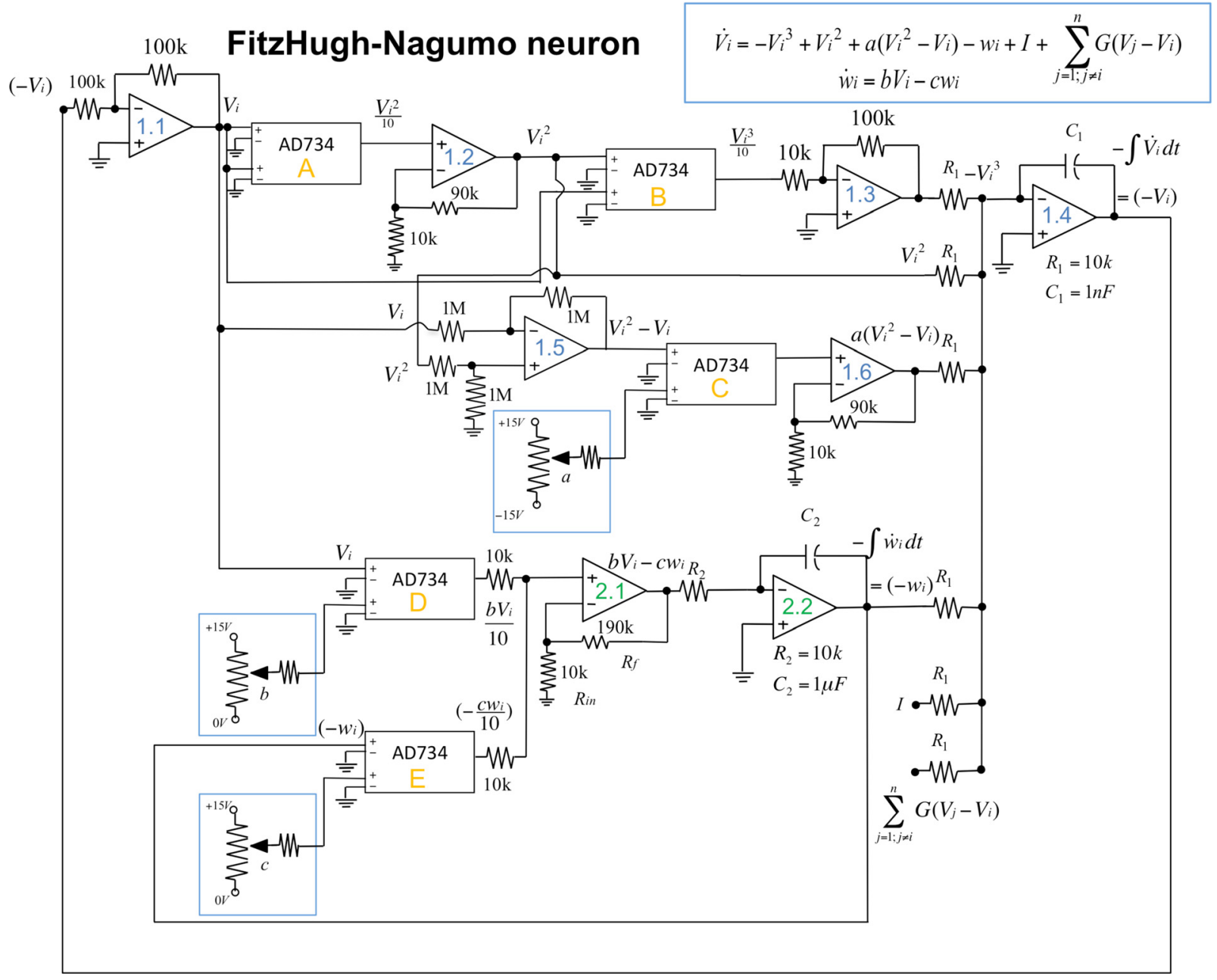

FIG. 2. Circuit diagram of electronic FitzHugh-Nagumo neuron. A, B, C, D, and E represent analog multipliers; 1.1, 1.2, 1.3, 1.4, 1.5, 1.6, 2.1, and 2.2 are quad op-amps; the time constants $\tau_{\mathrm{V}}=10 \mu \mathrm{s}$ and $\tau_{\mathrm{w}}=10 \mathrm{~ms}$ were chosen to yield a $3 \mathrm{~ms}$ refractory period. 
resulting $V^{2}$ and the original $V$ are input to another AD734 multiplier (B) to obtain $V^{3} / 10$, which is then rescaled by a factor of -10 to obtain $-V^{3}$ at the output of op amp (1.3). This is the first term on the right hand side of the $V$ equation. In this way each term of the equation can be built up. Figure 2 has been labeled to show the values of $V$ or $w$ at each stage of the circuit.

Since these are first-order differential equations, we need to integrate the terms on the right hand side once they have been added together to form $\dot{V}_{i}$. Op amp (1.4) performs this integration because the feedback around it is accomplished with a capacitor rather than a resistor. The output is $-V_{i}$.

The use of capacitors has another implication as well: time constants are introduced into the neuron. The integrating op amp (1.4) has a time constant $\tau_{V}$ of $10 \mu$ s and op amp (2.2) has a time constant $\tau_{w}$ of $10 \mathrm{~ms}$. These were chosen to mimic a real neuron having a refractory period of approximately $3 \mathrm{~ms}^{12}$

In similar fashion, the $w$ equation can also be constructed, as shown in the lower half of the figure. The constants $a, b$, and $c$ are set by dividing a voltage $(-15$ to $+15 \mathrm{~V}$ in the case of $a, 0$ to $15 \mathrm{~V}$ in the case of $b$ and $c$ ) via potentiometers.

The parameters $b$ and $c$, and the coupling strength $G$ were set using a programmable digital potentiometer, the Analog Devices 7376 . This is a 128-position, digitally controlled variable resistor controlled by a National Instruments USB 6289 Multifunction Data Acquisition device (DAQ). Both neuronal variables were sampled at $10 \mathrm{kHz}$ using the 18-bit analog inputs on the same device. National Instruments' LabviEw program controlled the DAQ to vary $b$ and $c$, and the coupling parameter $G$. A series resistor was added before each AD7376 to increase the resolution of the parameter $G$. A mechanical potentiometer was used to vary the parameter $a$.

Essential parts of the construction are the long lines running along the bottom from the output of op amp (1.4) to the input of op amp (1.1) as well as the line above it running from op amp (2.2) to the input of analog multiplier (E). These connect the output voltages to the input voltages and implement the "equal signs" in the $V$ and the $w$ equations, respectively.

External inputs such as $I$ and/or coupling to other neurons is done via the "hanging leads" at the lower right of the figure.

The neurons were weakly coupled by an op-amp configured as a subtractor and a digital potentiometer to vary the coupling strength between the neurons. Figure 1(b) shows how the voltage of two neurons $V_{i}$ and $V_{j}$ is subtracted and then multiplied by the coupling parameter $(G)$. Isolation amplifiers were inserted so that, while all neurons share the same parameter value, no crosstalk from one neuron to another is allowed through the parameter circuitry.

Figure 1(a) depicts the initial connection topology in green, with an optional nonlocal link shown in red. The coupling value, $G=0.0087$, is in the weak coupling limit. ${ }^{13}$ Note that the coupling strength of the additional link $G^{\prime}$ is one and a half orders of magnitude weaker than the nearest neighbor coupling, $G$.

\section{RESULTS}

The parameters for each neuron were individually selected to place the neuron approximately at threshold, the point at which spontaneous firing begins. This individual treatment is required because these are actual physical objects; imperfections and variances are inherent in the electronic components. Data were taken on each neuron individually and then the neurons were coupled into the ring topology.

Synchronization arises as the appearance of a relation between phases and frequencies. To find the instantaneous phase of the data, we reconstructed the phase of the signal via the Hilbert transform ${ }^{14}$

$$
\varsigma(t)=V(t)+j V_{H}(t)=A(t) e^{j \varphi(t)},
$$

where $V(t)$ is voltage and $V_{H}(t)$ is its transform. (Here, $j$ denotes the imaginary number and should not be confused with the index $j$ used previously.) $A(t)$ and $\varphi(t)$ are the resulting amplitude and phase, respectively. ${ }^{14}$

For the array of neurons with only nearest neighbor coupling, the array was unsynchronized when the coupling $G$ was zero. As $G$ was increased to about 0.01 , the neurons synchronized, with increasing $G$ leading to a decreasing spread in the phase angles of the neurons.

Now consider the case where a single long-range connection is added. This arrangement, known as small-world coupling ${ }^{15}$ radically changes the behavior of the network. Starting from a completely desynchronized state, as the coupling parameter $G$ was increased, some of the oscillators synchronized; while the rest synchronized into a separate group that was desynchronized from the first. Individual neurons would remain with their group for a while, but would then move into synchronization with the second group. Migration from the second group to the first also occurred. This was not a transitory effect, but rather was stable over the length of the experiment and is a hallmark of a chimera. $^{2-5}$ Examples of this phase clustering are shown for specific times in Fig. 3.

A much better understanding of the dynamics of the array can be gleaned from the movies included in the supplementary material. ${ }^{16}$ The movies are presented in temporal order, covering the frames (times) 3500 to 4600 , frames 9600 to 10100 , frames 1100300 to 1101500 , and finally frames 2003000 to 2004000 .

The actual circuit boards are also presented in the supplementary material. ${ }^{16}$ In this picture, a single neuron is outlined in the upper center. The circuitry for the parameters $a$, $b$, and $c$ is outlined in the lower left of the picture and the coupling circuitry $(G)$ is outlined in the lower right. The "untidiness" of the longer wiring serves to minimize stray capacitive coupling.

We have calculated the order parameter of the array in the standard fashion

$$
O(t)=\frac{1}{N} \sum_{k=1}^{N} e^{j \phi_{k}(t)}
$$

where $O(t)$ is the order parameter at time $t$ (in units of frame number), $\phi_{k}(t)$ is the phase of oscillator $k$ at time $t$, and $N=9$ 

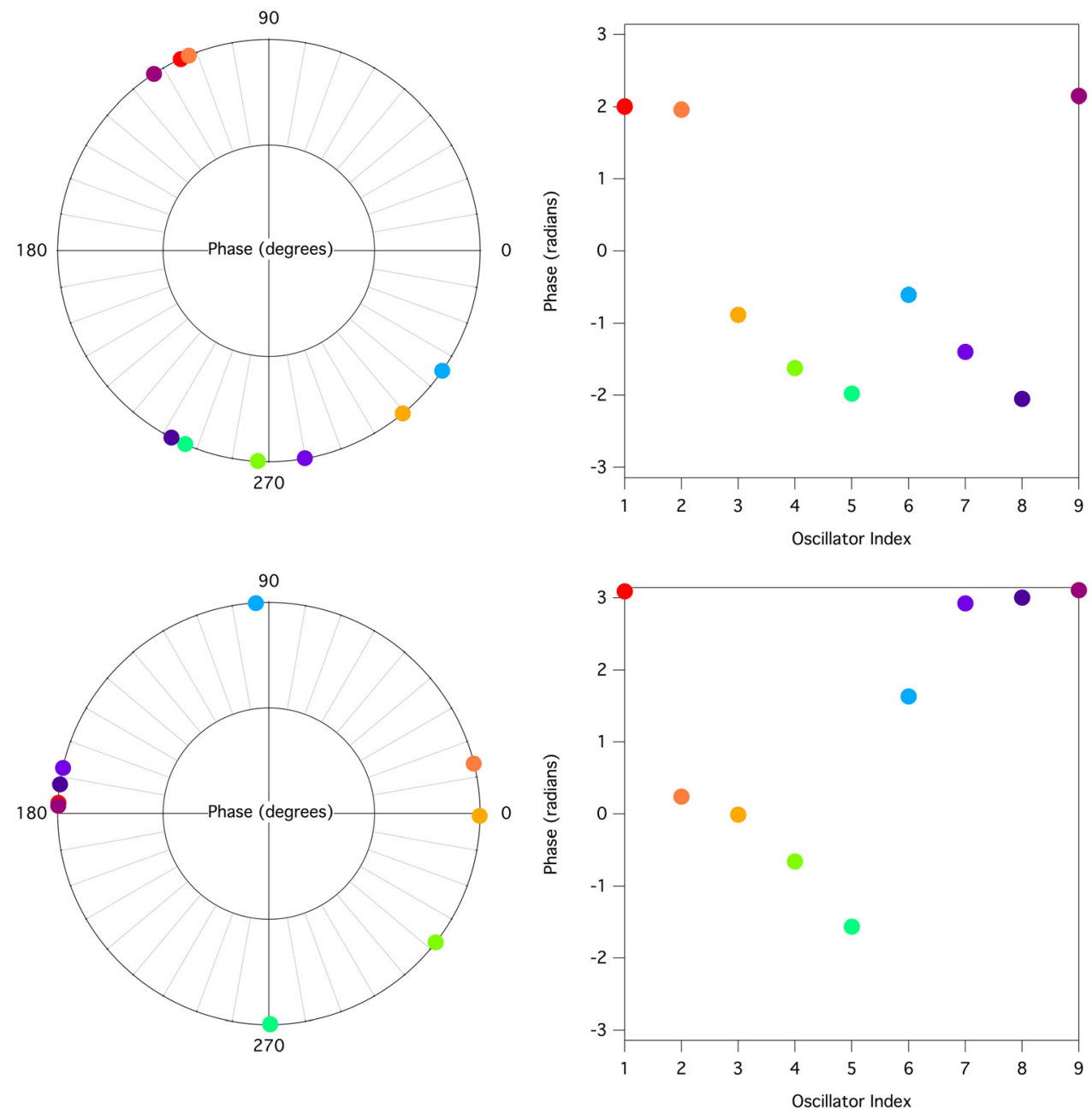

FIG. 3. Synchronization phase plots. (Left) Polar plots showing the phases (in degrees) of all oscillators at two different instants of time. (Right) Instantaneous spatial distribution of the corresponding phases of the oscillators in radians. Note that in the top frame, neurons 9,1 , and 2 are synchronized, while the rest are form a separately synchronized group. In the bottom frame, neurons $6,7,8,9$, and 1 are synchronized. Neuron 6 is caught in a transition from one group to another. The parameters and the coupling constants are the same as in Fig. 1.

is the total number of oscillators. Among other things, the order parameter is a good indicator of when transients due to experimental startup have dissipated. Fig. 4 shows a plot of the magnitude of order parameter as a function of coupling.
Unsurprisingly, this run-averaged order increases as the nearest neighbor coupling increases. Of more interest is the variation in the order parameter as a function of time (Fig. 4, right). The order varies in a complex fashion, with occasional large
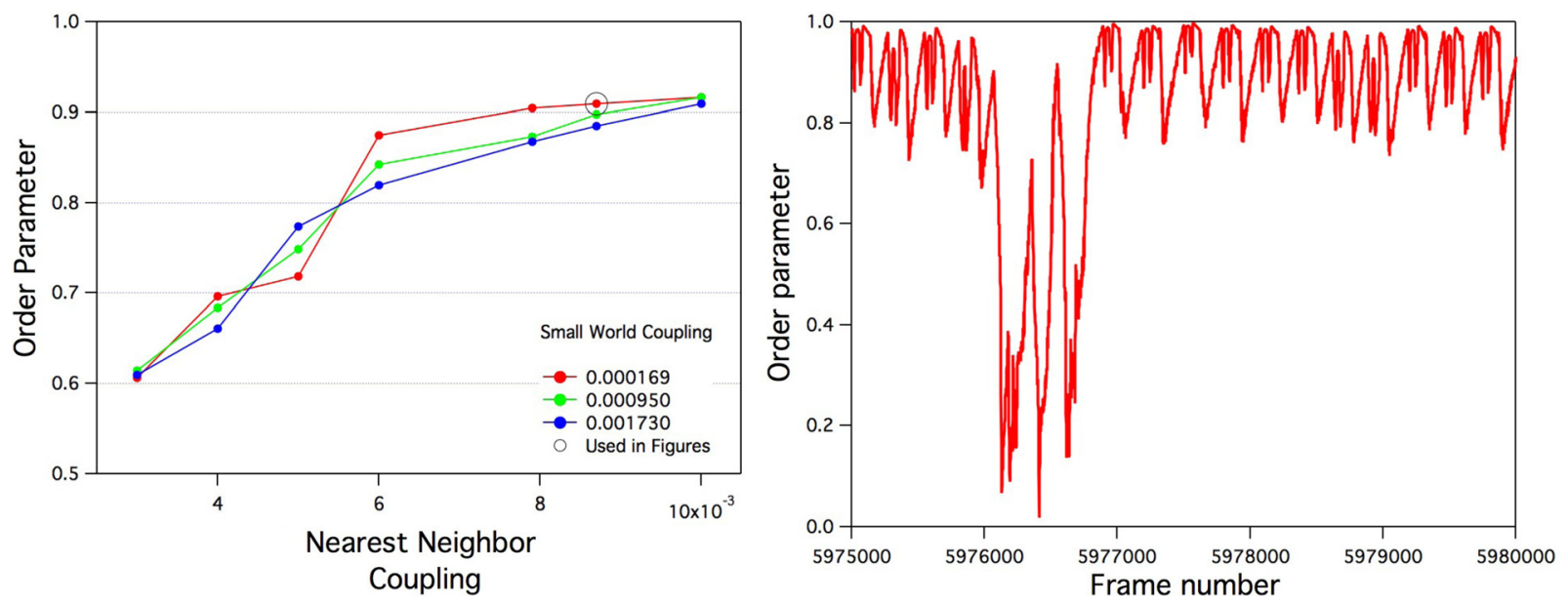

FIG. 4. (Left) Order parameter (averaged over the entire length of each run) as a function of the nearest neighbor coupling. Different values of the small world coupling did not significantly affect the order. (Right) Expanded view of the parameter at the coupling values denoted by the circle in the left pane. 
excursions from order to disorder and back whenever an individual oscillator migrates from one group to another. We speculate that the smaller, more regular excursions represent internal modes of the group of oscillators.

Kuramoto and Battogtokh ${ }^{2}$ first theorized the existence of chimeras when studying non-locally coupled systems. Since the first experimental observations of chimeras, one in an optical system by Hagerstrom et al. ${ }^{4}$ as well as in chemical oscillators by Tinsley et al., ${ }^{5}$ there have been many other papers and reviews on the subject.

Dudkowski et al. ${ }^{17}$ have begun to classify the different types of chimera states observed in the literature. However, they consider either maps or a driven system (the Van der Pol oscillator), while our system is both experimental and undriven. Their techniques are difficult to apply to a noisy experimental system.

Panaggio and Abrams ${ }^{18}$ have done a systematic review of the field and have collected several references on "bumpstates" in neural networks. However, the observation of chimeras in such networks has not been reported until Gambuzza ${ }^{11}$ and the current work.

\section{CONCLUSIONS}

We have already mentioned the paper by Gambuzza et al. ${ }^{11}$ Their results differ from ours in that we do not see chimeras unless small world coupling is introduced. This difference may be due to the radically different types of coupling employed. However, both papers have shown that chimeras can be seen in neuronal systems, but there seems to be a complex and sensitive dependence on the type and range of the coupling.

We report three primary results. First, the introduction of a single, weak long-distance connection $G^{\prime}$ can transform the behavior of the neuronal network as a whole from all-ornothing synchronization to a situation where chimeric behavior arises. That this transformation can occur in an array with neurons that are "normal" and roughly identical suggests that the synchronization seen in interictal spikes may be due to the topology of the interneuron connections and need not involve any pathology of the individual neurons themselves.

Second, the process of neuronal synchronization seen during the development of interictal spikes prior to epileptiform behavior need not be an "all or nothing" affair. Rather, we show that the synchronization may proceed through intermediate (chimeric) stages. This has implications both for the prediction of seizures as well as for possible electrical interventions.

Speculatively, grand mal seizures (or tonic-clonic seizures) are a class of seizure in which discharges involve synchronous high frequency neuronal firing propagating through long-range connections in the nervous system. ${ }^{19}$ The corpus callosum link between the left and right hemispheres of the brain passes neuronal signals back and forth between the hemispheres in a positive feedback loop, causing this electrical activity. Synchrony plus the long-range link requires the consideration of a chimeric approach to that synchrony.

Third, the FitzHugh-Nagumo neuronal model is generic. Thus, the ideas presented here may apply whenever the synchronization of neurons is relevant, such as in the perception of a signal by sensory neurons. Thus, it could be important for information processing, where coordination (more appropriate in this context than the term synchronization) of neurons, whether in the same region of the body or in different regions (recall the long-range connection above) is important.

The current study suggests the need to examine the relevance of chimeras in vivo. Studies of synchronization in split brain could be done for generalized tonic-clonic epileptic patients, whose corpus callosum has been removed in order to stop signal propagation between the hemispheres.

In conclusion, the synchrony seen in interictal spikes need not be an "all or nothing" event. There may be intermediate stages through which the process of neuronal synchronization occurs. The chimera state that we have demonstrated in our neuronal system is an example of such an intermediate stage, where synchronization and desynchronization coexist in the same system.

\section{ACKNOWLEDGMENTS}

We thank M. Shlesinger for helpful discussions and we gratefully acknowledge support from the U.S. Office of Naval Research under Grant No. N00014-12-1-0320.

${ }^{1}$ K. J. Staley and F. E. Dudek, Epilepsy Curr. 6, 199 (2006).

${ }^{2}$ Y. Kuramoto and D. Battogtokh, Nonlinear Phenom. Complex Syst. 5, 380 (2002).

${ }^{3}$ D. M. Abrams and S. H. Strogatz, Phys. Rev. Lett. 93, 174102 (2004).

${ }^{4}$ A. M. Hagerstrom, T. E. Murphy, R. Roy, P. Hövel, I. Omelchenko, and E. Schöll, Nat. Phys. 8, 658 (2012).

${ }^{5}$ M. R. Tinsley, S. Nkomo, and K. Showalter, Nat. Phys. 8, 662 (2012).

${ }^{6}$ R. FitzHugh, Biophys. J. 1, 445 (1961).

${ }^{7}$ J. Nagumo, S. Arimoto, and S. Yoshizawa, Proc. IRE 50, 2061 (1962).

${ }^{8}$ A. L. Hodgkin and A. F. Huxley, J. Physiol. 117, 500-544 (1952).

${ }^{9}$ E. M. Izhikevich, IEEE Trans. Neural Networks 15, 1063 (2004).

${ }^{10}$ E. M. Izhikevich, Dynamical Systems in Neuroscience: The Geometry of Excitability and Bursting (MIT Press, Cambridge, MA, 2007), p. 106.

${ }^{11}$ L. V. Gambuzza, A. Buscarino, S. Chessari, L. Fortuna, R. Meucci, and M. Frasca, Phys. Rev. E 90, 032905 (2014).

${ }^{12}$ F. Moss, J. K. Douglass, L. Wilkens, D. Pierson, and E. Pantazelou, Ann. N. Y. Acad. Sci. 706, 26 (1993).

${ }^{13}$ A. Pikovsky, M. Rosenblum, and J. Kurths, Synchronization: A Universal Concept in Nonlinear Sciences (Cambridge University Press, Cambridge, 2001), p. 16.

${ }^{14}$ A. Pikovsky, M. Rosenblum, and J. Kurths, Synchronization: A Universal Concept in Nonlinear Sciences (Cambridge University Press, Cambridge, 2001), p. 362.

${ }^{15}$ D. J. Watts and S. H. Strogatz, Nature 393, 440 (1998).

${ }^{16}$ See supplementary material at http://dx.doi.org/10.1063/1.4905856 for synchronization phase plot movies. On the left is a polar plot showing the phases (in degrees) of all oscillators. The right plot displays the spatial distribution of the corresponding phases of the oscillators in radians. Supplementary movie S1 covers the time period from frame 3500 to 4600. Supplementary movie S2 covers the time period from frame 9600 to 10100 . Supplementary movie S3 covers the time period from frame 1100300 to 1101500 . Supplementary movie S4 covers the time period from frame 2003000 to 2004000 . We also include in the supplementary material a picture of the circuit board for the entire neuronal array.

${ }^{17}$ D. Dudkowski, Y. Maistrenko, and T. Kapitaniak, Phys. Rev. E 90, 032920 (2014).

${ }^{18}$ M. J. Panaggio and D. M. Abrams, "Chimera states: Coexistence of coherence and incoherence in networks of coupled oscillators," e-print arXiv:14036204 (2014).

${ }^{19}$ Y. Zhong, G. Lu, Z. Zhang, Q. Jiao, K. Li, and Y. Liu, Epilepsy Res. 97, 83 (2011). 